Psychotherapeut 2013 · 58:325-326

DOI 10.1007/s00278-013-0994-4

Online publiziert: 11. Juli 2013

(c) Springer-Verlag Berlin Heidelberg 2013
Dorothea von Haebler ${ }^{1,2} \cdot$ Harald Freyberger $^{3}$

${ }^{1}$ Klinik für Psychiatrie und Psychotherapie, Charité-Universitätsmedizin Berlin, Campus Mitte, Berlin

${ }^{2}$ International Psychoanalytic University Berlin

${ }^{3}$ Klinik für Psychiatrie und Psychotherapie, Universitätsmedizin Greifswald

\title{
Psychotherapie für Menschen mit Psychosen ist möglich
}

Das vorliegende Heft der Zeitschrift Psychotherapeut beschäftigt sich mit „schweren psychischen Störungen“. Die im englischen Sprachraum als ,severe mentally ill“ bezeichneten Gruppen definieren sich u. a. durch die Behandlungshäufigkeit (u. a. „heavy user"), und es handelt sich in den meisten Fällen um psychotisch erkrankte Menschen. Diese kommen zwar häufiger als andere psychisch Kranke mit dem Gesundheitssystem in Berührung, aber viel zu selten kommt es zu einer tragfähigen therapeutischen Beziehung. Dies aus ihrer Krankheit heraus zu erklären, ist eine Perspektive. Mindestens genauso wichtig erscheint es jedoch, die bestehenden Versorgungsstrukturen dafür verantwortlich zu machen. Die aktuellen Veränderungen in der psychiatrischen Versorgungslandschaft, wie die integrierte Versorgung, machen Hoffnung, dass spezifische Formen für die Therapie dieser Gruppen implementiert werden können und damit selbstverständlicher werden: So ist $z$. B. die aktuell praktizierte „Kommstruktur“ der Psychiatrie für Menschen, die große Schwierigkeiten haben, in Beziehung zu treten, kein Angebot, sondern häufig eine Zumutung.

Die psychotherapeutische Behandlung erfolgt in Deutschland im Wesentlichen über die Richtlinienpsychotherapie, und hier zeigen Untersuchungen, dass über $90 \%$ der Psychotherapien für affektive und im weitesten Sinn neurotische Störungen genehmigt werden, während Erkrankungen aus dem schizophrenen Formenkreis dort mit weniger als $1 \%$ repräsentiert sind.
Die Psychotherapie bei psychotischen Erkrankungen ist eine von den meisten Professionellen und Betroffenen akzeptierte und effektive Behandlungsmethode, die durchaus in den Behandlungsplänen vorkommt. Allerdings kommt sie insbesondere im ambulanten System selten zur Anwendung, da neben einer notwendigen Modifikation der Verfahren auch die Unterstützung des Therapeuten im Hinblick auf krisenhafte Zuspitzungen, Ausbildung und Vergütung gewährleistet sein muss.

Was macht die Therapie von Menschen aus, die um zu überleben, den Boden der Realität verlassen, uns als Therapeuten möglichst wenig Zugriff, aber viel Anstoß geben, und dabei äußerst sensibel für jede Form von Signalen sind?

Es geht um eine sehr offene Haltung, eine Offenheit für das, was das Gegenüber so bewegt, in die Enge getrieben hat, einen Raum, der zulässt, dass ein Ausweg gefunden wird, eine große Geduld, um Platz zur Überwindung von Ambivalenzen zu schaffen und bei allem dennoch ein aktives Nachgehen, wenn die Zielgerichtetheit des Gegenübers nicht ausreicht oder schädigend wird. In solch einer Atmosphäre ist es besonders wichtig, immer wieder den perspektivischen Raum zu erweitern: Supervision, Intervision und Reflexion sind bei Psychosenpsychotherapie unabdingbar. Dann kann die Bereitschaft, sich auf unerwartete Entwicklungen einzulassen, ein Prozess werden, in dem nicht die Angst (des Patienten und des Therapeuten) die Richtung vorgibt, sondern anstelle dessen neue, individuelle Wege entste- hen, die z. T. vom Patienten und z. T. vom Psychotherapeuten stammen.

In diesem Heft werden vor diesem Hintergrund neue Konzepte und die Weiterentwicklung bestehender Konzepte dargestellt:

Der Beitrag von Lempa et al. stellt eine Weiterentwicklung des vor wenigen Monaten veröffentlichten Werkzeugkastens dar. Ziel des Autorenteams ist es, ein Manual der psychodynamischen Psychotherapie für Menschen mit Psychosen zu entwickeln, das hilft, das modifizierte Verfahren zu verbreiten und schließlich auch zu beforschen. Das Beschreiben von konkreten Problemfeldern in der Begegnung des Therapeuten mit dem Patienten und der einzusetzenden Werkzeuge wird anhand einer Fülle von konkreten Fallbeispielen vorgenommen.

Ein neues Konzept ist z. B. die mentalisierungsbasierte Psychotherapie für Menschen mit Psychosen. Diese Therapieform wurde ursprünglich für Patienten mit emotional instabilen Persönlichkeitsstörungen entwickelt. Sachs u. Felsberger haben diese Psychotherapiemethode für Menschen mit Psychosen adaptiert und legen hierzu ein Konzept vor, dessen störungsbezogene Interventionen in der stationären Gruppenpsychotherapie praktiziert und mit guten Ergebnissen evaluiert wurden.

Weiterentwickelt wurde auch Psychotherapie in der Früherkennung. Müller u. Bechdolf referieren über verschiedene methodische Aspekte, fassen die Studienlage zusammen und kommen zu dem Schluss, dass kognitive Verhaltensthera- 
pie (KVT) die beste Evidenz für die psychotherapeutische Behandlung von Menschen mit erhöhtem Psychoserisiko hat. Insbesondere in der Früherkennung ist es notwendig, auch die Nebenwirkungen eines psychotherapeutischen Verfahrens mitzubetrachten.

Klingberg $u$. Hesse beschreiben in ihrem Beitrag über kognitive Verhaltenstherapie für Menschen mit Psychosen zunächst die gute Studienlage und schließlich die Notwendigkeit, auch diesen Ansatz zu modifizieren. Neben der Aufgabe einer individuellen Therapieplanung wird wie bei dem Beitrag von Bechdolf et al. auf die dringende Empfehlung zur Integration von Angehörigen in den therapeutischen Prozess hingewiesen.

Schweitzer u. Aderhold berichten über die systemische Therapie bei Menschen mit Psychosen, die im Wesentlichen im skandinavischen Raum weiterentwickelt wurde. Die Zentren, in denen diese Form der Psychotherapie praktiziert wird, werden genannt.

Es folgt ein Beitrag von Bock et al. zu Peer-Beratung und Psychosenpsychotherapie. Die von ihnen beschriebene PeerBeratung ist in Deutschland eine neue Methode, die auf eine andere Rolle der Erkrankten im Gesundheitssystem hinweist und die es deshalb besonders verdient, dargestellt zu werden. Nicht vielen in der Versorgung tätigen Psychotherapeuten ist diese Entwicklung bekannt, die eine völlig neue therapeutische Beziehung definiert.

Zur psychotherapeutischen Behandlung von Menschen mit Psychosen ist, und das ist in den Beiträgen des vorliegenden Hefts der Zeitschrift Psychotherapeut eindrücklich beschrieben, eine Modifikation gängiger Verfahren und Methoden notwendig. Aus allen Beiträgen ist der erhebliche Forschungsbedarf abzuleiten, der nicht nur Studien zur Effektivität und Effizienz einschließt, sondern sich auch mit den speziellen Implementierungshindernissen beschäftigen sollte.

\section{Korrespondenzadresse}

\section{Dr. Dorothea von Haebler}

Klinik für Psychiatrie und Psychotherapie,

Charité-Universitätsmedizin Berlin,

Campus Mitte

Charitéplatz 1, 10117 Berlin

dorothea.vonhaebler@charite.de

\section{Einhaltung der ethischen Richtlinien}

Interessenkonflikt. D. von Haebler und H. Freyberger geben an, dass kein Interessenkonflikt besteht.

Das vorliegende Manuskript enthält keine Studien an Menschen oder Tieren.

\section{Schwerpunktthemen}

Das Herausgebergremium der Zeitschrift Psychotherapeut lädt Autorinnen und Autoren ein, an den geplanten Schwerpunkten mitzuarbeiten und geeignete Manuskripte einzureichen. Diese werden dem üblichen Reviewverfahren unterzogen. Darüber hinaus freuen wir uns über die Zusendung freier (unaufgeforderter) Originalia zu selbstgewählten Themen.

Bitte schicken Sie Ihren Beitrag an die Redaktion:

Regine.Karcher-Reiners@springer.com

Die Schwerpunktplanung ist vorläufig und kann kurzfristig umdisponiert werden.

Heft 1/14: Psychotherapie adoleszenter Patienten mit Gewalt- und Traumaerfahrungen (Deadline 30.08.2013)

Heft 2/14: Kulturwandel in der Psychotherapie (Deadline 30.10.2013)

Heft 3/14: Integrations-Kultur in der Psychotherapiee

(Deadline 30.12.2013)

Heft 4/14: Stigma und Stigmatisierung

(Deadline 30.02.2014) 